Canum 2000 : Actes du 32e Congrès national d'analyse numérique

A. Blouza, I. Danaila, P. Joly, S.M. Kaber, B. Lucquin, F. Murat \& M. Postel, Éditeurs

ESAIM: Proceedings, Vol. 11, 2002, 13-40

http://www.emath.fr/Maths/Proc/Vol.11/

(C)2002, Société de Mathématiques Appliquées et Industrielles, EDP Sciences

\title{
An Eulerian numerical method for geometric optics
}

\author{
Jean-David Benamou
}

\begin{abstract}
Résumé. La description classique du problème de l'optique géométrique est Lagrangienne. Le passage à la représentation Eulérienne est possible mais, en présence de caustiques, ne permet pas de calculer la solution Lagrangienne complète. On présente le contexte mathématique du problème ainsi que quelques outils numériques développés pour lever cette limitation.
\end{abstract}

Mots clés. Hamilton-Jacobi, caustique, système Hamiltonien, lancer de rayons, solution de viscosité

\begin{abstract}
The classical description for geometric optics is Lagrangian. It is possible to switch to an Eulerian representation but, in presence of caustics, the full Lagrangian solution cannot be computed using a Eulerian method. We present the mathematical background of the problem and several numerical tools developed to overcome this limitation.
\end{abstract}

Key words. Hamilton-Jacobi, Caustic, Hamiltonian System, Ray Tracing, Viscosity Solution

AMS subject classification. 49L25, 70H05, 70H20, 78A05

\section{Introduction}

Ray tracing is a widespread numerical technique. Its is routinely used for instance in the oil industry to compute travel-times of seismic waves in the underground under the geometric optics approximation for high frequency wave propagation. Geometric optics is also a prototype problem for first order Hamilton-Jacobi equations where the Hamiltonian function is convex. This class of problems appears in rather diverse applications such as wave propagation, mesh generation, combustion, crystal growth and more generally in a large number of problems of the calculus of variations.

Ray tracing consists in solving a set of ordinary differential equations called the Hamiltonian system. One of the variable describes a Lagrangian trajectory (the ray) along which the others, the Lagrangian variables, are transported. This approach is conceptually simple and numerically easy to use. As a Lagrangian method however it does not give any control on the spatial resolution of the Lagrangian solution. Indeed, the rays are necessarily in finite numbers and produce when diverging poor resolution (a Lagrangian attempt to solve this problem is the Wavefront 
Construction method [35] [26]) . When converging (the opposite phenomena), the rays can cross and caustics appear. In this case the Lagrangian trajectories naturally sweep several times the same space location where the Lagrangian variables may take different values. The Lagrangian solution is in this sense multi-valued.

When switching to Eulerian variables defined on the configuration space, we obtain an Hamilton-Jacobi partial differential equation. It can be discretized on a fixed grid which in particular does not depend on the rays. The space resolution of the numerical method is therefore easily maintained and the accuracy of the approximation depends on this resolution. In the classical theory however, these two approaches, Lagrangian and Eulerian, are equivalent only where the Lagrangian solution is well defined and single-valued.

The problem of computing the Lagrangian solution using a Eulerian representation when it is multi-valued has been the subject of on-going work in the last half decade ([31] [18] [7] [2] [29] [14] [19] [8] [24] ...). Most of these studies were motivated by potential applications to high frequency wave propagation problem (see for instance [25] [27] [17] [21]) for which the exact model is numerically intractable sometimes even in 2-D. See also [11] on this application in presence of a cuspidal caustic which will be our main topic of interest.

We first review here the mathematical framework of the problem, introducing both Lagrangian and Eulerian "viscosity" solutions and their relations (sections 1 to 3). The structure of the multi-valued solution associated to a cuspidal caustic is detailed on a particular example (section 4) and used in a second part to illustrate the successive numerical Eulerian algorithms proposed to solve the problem in the general case (section 5). Numerical results are presented in the third part (section 6).

\section{The Lagrangian solution and the ray method}

\subsection{The Hamiltonian system}

A Hamiltonian function $H(t, y, p)$ is given, defined on $\mathbb{R}_{s}^{+} \times \mathbb{R}_{y}^{d} \times \mathbb{R}_{p}^{d}, d$ is the space dimension of the problem, $\mathbb{R}_{s}^{+} \times \mathbb{R}_{y}^{d}$ is the time-space configuration in which rays can evolve and $\mathbb{R}_{s}^{+} \times \mathbb{R}_{y}^{d} \times \mathbb{R}_{p}^{d}$ is called the phase space. The Hamiltonian is assumed to be continuous up to its second derivatives and convex and coercive in its last variable $p$.

The Lagrangian method then consists in solving the Hamiltonian system formed by the following set of ordinary differential equations (ODEs) [36] [3] [23] [17] :

$$
\left\{\begin{array}{l}
\dot{y}\left(s, y^{0}\right)=H_{p}\left(s, y\left(s, y^{0}\right), p\left(s, y^{0}\right)\right), y\left(0, y^{0}\right)=y^{0} \\
\dot{p}\left(s, y^{0}\right)=-H_{y}\left(s, y\left(s, y^{0}\right), p\left(s, y^{0}\right)\right), p\left(0, y^{0}\right)=\phi_{y^{0}}^{0}\left(y^{0}\right), \\
\dot{\varphi}\left(s, y^{0}\right)=p\left(s, y^{0}\right) \cdot H_{p}\left(s, y\left(s, y^{0}\right), p\left(s, y^{0}\right)\right)-H\left(s, y\left(s, y^{0}\right), p\left(s, y^{0}\right)\right), \\
\varphi\left(0, y^{0}\right)=\phi^{0}\left(y^{0}\right) .
\end{array}\right.
$$

ESAIM: Proc., Vol. 11, 2002, 13-40 
The dot stands for "time" derivation $()=.\frac{d(.)}{d s}, g_{x_{i}}\left(x_{1}, x_{2}\right)$ and $g_{x_{i} x_{j}}\left(x_{1}, x_{2}\right)$ respectively denote the gradient and the Hessian of $g$ with respect to $x_{i}$ and $\left(x_{i}, x_{j}\right), \phi^{0}$ is the initial phase and also appears in the initial condition for $p$. For each $y^{0} \in \mathbb{R}_{y}^{d}$ (or a subset of $\mathbb{R}_{y}^{d}$ ), the system generates bicharacteristics strips $\left(y\left(s, y^{0}\right), p\left(s, y^{0}\right)\right)$ lying in phase-space with smooth dependence on $s$ and $y^{0}$. The projections of the strips onto $\mathbb{R}_{y}: y\left(s, y^{0}\right)$, are called the rays. Each ray is therefore "labeled" by its initial position $y^{0}$. The phase $\varphi\left(s, y^{0}\right)$ is transported by the corresponding ray $y\left(s, y^{0}\right)$ and, when rays are crossing, is a multi-valued function of the configuration space $\mathbb{R}_{s}^{+} \times \mathbb{R}_{y}^{d}$.

It happens in particular at caustics which are the points on the rays where an infinitesimal tube of neighboring rays collapses. Mathematically, a ray $y\left(s, y^{0}\right)$ encounters a caustic point when the determinant (denoted |.|) of the Jacobian matrix of $y$ with respect to $y^{0}$ :

$$
\alpha\left(s, y^{0}\right)=\left|\frac{\partial y\left(s, y^{0}\right)}{\partial y^{0}}\right|
$$

is zero. The quantity $\alpha$ is sometimes called "geometric spreading" as it provides a local measure of the geometric convergence or divergence of the rays.

Caustics being an important ingredient of multi-valuedness and therefore of our problem, it is important to understand how $\alpha\left(s, y^{0}\right)$ can be evaluated. The computation of $\frac{\partial y\left(s, y^{0}\right)}{\partial y^{0}}$ is performed using a set of additional ODEs which are obtained via a linearization of the system (1) with respect to $y^{0}$ :

$$
\left\{\begin{array}{l}
\left(\begin{array}{l}
\frac{\dot{\partial} y}{\partial y^{0}}\left(s, y^{0}\right) \\
\frac{\dot{\partial} p}{\partial y^{0}}\left(s, y^{0}\right)
\end{array}\right)=A\left(s, y\left(s, y^{0}\right), p\left(s, y^{0}\right)\right) \cdot\left(\begin{array}{c}
\frac{\partial y}{\partial y^{0}}\left(s, y^{0}\right) \\
\frac{\partial p}{\partial y^{0}}\left(s, y^{0}\right)
\end{array}\right) \\
\left(\begin{array}{c}
\frac{\partial y}{\partial y^{0}}\left(0, y^{0}\right) \\
\frac{\partial p}{\partial y^{0}}\left(0, y^{0}\right)
\end{array}\right)=\left(\begin{array}{l}
I d_{d \times d} \\
\frac{\partial^{2} \phi^{0}}{\partial y^{0}}\left(y^{0}\right)
\end{array}\right)
\end{array}\right.
$$

where

$$
A(t, y, p)=\left(\begin{array}{cc}
H_{p y}(s, y, p) & H_{p p}(s, y, p) \\
-H_{y y}(s, y, p) & -H_{y p}(s, y, p)
\end{array}\right) .
$$

This is a set of $2 d^{2}$ ODEs but, as it is written, each unknown of system (3) is a $d \times d$ matrix and the matrix vector product must be understood in the correct algebra. Finding a caustic point on a ray therefore consists in solving (1-3) and evaluate $\alpha\left(s, y^{0}\right)$ along a ray using (2). When it vanishes or more precisely changes its sign, the ray has passed a caustic point.

\subsection{The Lagrangian sub-manifold and its singularities}

We would like now to know when the Lagrangian solution is multi-valued. It is of course impossible to access this information a priori ; but differential geometry provide some information on 
the possible structures of multi-valued solutions (see [5] [4] [17] [23]).

In this theory, the set of bicharacteristics strips $\Lambda=\left\{\left(s, y\left(s, y^{0}\right), p\left(s, y^{0}\right)\right) ;\left(s, y^{0}\right) \in \mathbb{R}_{s}^{+} \times\right.$ $\left.\mathbb{R}_{y}^{d}\right\}$ is considered as a (Lagrangian) sub-manifold of co-dimension $d$ of phase-space $\mathbb{R}_{s}^{+} \times \mathbb{R}_{y}^{d} \times \mathbb{R}_{p}^{d}$. Next we introduce the canonical projection from phase space to configuration space :

$$
\begin{aligned}
\Pi: \mathbb{R}_{s}^{+} \times \mathbb{R}_{y}^{d} \times \mathbb{R}_{p}^{d} & \longrightarrow \mathbb{R}_{s}^{+} \times \mathbb{R}_{y}^{d} \\
(s, y, p) & \longrightarrow(s, y) .
\end{aligned}
$$

When $\Lambda$ folds on itself in the $p$ dimension direction, the Lagrangian solution $\Pi(\Lambda)$ is multi-valued and the projection become singular on the fold. The projection of the fold is the caustic. Quite remarkably, differential geometry provide a generic classification of the different stable ways $\Lambda$ can locally fold (stable means here that the structure described below of $\Lambda$ cannot change under small perturbation in phase space). More precisely, up to an unknown change of variable in phase space which preserves the volume element $d y \wedge d p$, a local analytical description of $\Lambda$ near multi-valued solution is given. For $d=1$, which corresponds (with the "time" dimension) to a two dimensional problem, there are two possibilities where $(s, p)$ are used to parameterize $\Lambda$ : the Fold, $y=3 p^{2}$ and the Cusp, $y=4 p^{3}+2 s p$ (which corresponds two the junction of two Folds). A similar but larger classification exists for $d=2,3$.

The multi-valued geometric solution can, of course, be more complicated. Different foldings may interact or superimpose. However, locally, it can always be reduced to several elementary caustics. The purpose of the presentation is to describe a numerical method for computing the multi-valued solution associated to a general cuspidal caustic (which contains the case of the Fold as a sub-case). This building block could be used then in a general algorithm able to cope with caustic interactions. See [8] for more on that subject.

\section{The Eulerian solution and the Hamilton-Jacobi partial differential equation}

\subsection{From Lagrangian to Eulerian}

We assume that the following "transversality" condition

$$
\alpha\left(0, y^{0}\right) \neq 0
$$

holds. Then, as we proceed along the ray and as long as the "no caustic" property $\alpha\left(s, y^{0}\right) \neq 0$ is satisfied, we can apply the local inversion theorem to the mapping $y^{0} \rightarrow y\left(s, y^{0}\right)$. This implicitly means that the Eulerian variable $\phi(s, x)$ evaluated at the Lagrangian coordinates specified by the rays matches the Lagrangian phase :

$$
\phi\left(s, y\left(s, y^{0}\right)\right)=\varphi\left(s, y^{0}\right) .
$$

ESAIM: Proc., Vol. 11, 2002, 13-40 
It is well defined in the domain spanned by the rays $y\left(s, y^{0}\right)$ such that $\alpha\left(s, y^{0}\right) \neq 0$. This is equivalent to a no caustic assumption.

Note that at time 0 the gradient of $\phi\left(0, y^{0}\right): \phi_{x}\left(0, y^{0}\right)$ is, by definition, equal to $p\left(0, y^{0}\right)$. A classical result of the calculus of variation [22] [36] states that this property is preserved, again as long as $\alpha\left(s, y^{0}\right) \neq 0$ :

$$
\phi_{x}\left(s, y\left(s, y^{0}\right)\right)=p\left(s, y^{0}\right) .
$$

It is then straightforward to derive the Hamilton-Jacobi equation, first in Lagrangian coordinates and then back to Eulerian coordinates :

$$
\left\{\begin{array}{l}
\frac{\partial \phi}{\partial s}(s, x)+H\left(s, x, \phi_{x}(s, x)\right)=0, \text { for }(s, x) \in \mathbb{R}_{s}^{+} \times \mathbb{R}_{y}^{d} \\
\phi\left(0, y^{0}\right)=\phi^{0}\left(y^{0}\right), \text { for } y^{0} \in \mathbb{R}_{y}^{d} .
\end{array}\right.
$$

When the above conditions on $\alpha$ are satisfied, $\phi$ defined by (6) is called a classical solution of (8).

\subsection{The viscosity solution}

When the conditions for a classical solution are not satisfied, there is still a notion of global weak solution for equation (8) called "viscosity solution" [15] [6]. Viscosity solutions are the correct objects to consider because any stable numerical scheme converges to this class of solution [30] [16]. These schemes are generally called upwind because they discretize space derivatives on the opposite side to the direction of the rays (would rays be traced).

A link can be made between Lagrangian and Eulerian viscosity solution using the theory of optimal control [8]. The viscosity solution can be characterized as the value function of the following optimization problem

$$
\phi(s, x)=\underset{\substack{\left\{y^{0} \in \mathbb{R}_{y}, y(.) \in W^{1,+\infty}(\mathbb{R}) ; \\ y(0)=y^{0}, y(s)=x\right\}}}{\int_{0}^{s} L(t, y(t), \dot{y}(t)) d t+\phi^{0}\left(y^{0}\right),}
$$

where the minimization is performed with respect to the admissible curves $y($.$) and their initial$ point $y^{0}$. The "Lagrangian" function $L(s, x, v)=\sup _{p \in \mathbb{R}_{p}}\{p \cdot v-H(s, x, p)\}$ is the Legendre transform of $H$ with respect to $p$. One can formally check that the Euler-Lagrange equations of this problem correspond to the Hamiltonian system (1). It means that the rays are the critical curves of the optimization problem (9) and the viscosity solution the optimal value of the cost function.

When each point is only reached by one ray, we have a single-valued classical solution and the value function of problem (9) is exactly the integral of the phase ODE in (1).

If more than one ray reaches the time space point $(s, x)$ and if we denote $\left(y_{k}^{0}\right)_{k=1 . . n}$ the $n$ initial points of these curves, then the viscosity solution $\phi$ selects minimum of the associated phases :

$$
\phi(s, x)=\min _{k=1 . . n} \varphi\left(s, y_{k}^{0}\right)
$$

ESAIM: Proc., Vol. 11, 2002, 13-40 
If there is a zone where no ray penetrates, the viscosity solution implicitly generates "nonclassical" rays to fill this empty zone. It means that the optimal curves will satisfy the Hamiltonian system (1) with initial conditions different from what is specified for classical rays. See [10] for more on this phenomena. When the configuration space is bounded, the optimal curves may only satisfy the ray equations (1) piecewise (they can be reflected or diffracted) or they can creep along boundaries. These interesting situations are probably linked to diffraction phenomena and should hopefully be investigated elsewhere.

\subsection{Transport equations}

Of course (8) alone only provides the phase. As in the Lagrangian method we can introduce additional equations to determine caustics. Let $\delta(s, x)$ be a new Eulerian vector valued variable $\delta(s, x)$ configured in time-space $(s, x) \in \mathbb{R}_{s}^{+} \times \mathbb{R}_{y}^{d}$ and defined by $\left({ }^{T}\right.$ is the transpose of .) :

$$
\delta\left(s, y\left(s, y^{0}\right)\right)=\left(\frac{\partial y\left(s, y^{0}\right)}{\partial y^{0}}, \frac{\partial p\left(s, y^{0}\right)}{\partial y^{0}}\right)^{T} .
$$

Like for the Hamilton-Jacobi (see section 4.3), each component of $\delta(t, x), \delta_{i}(t, x)$ can be shown to satisfy a Eulerian transport equation :

$$
\left\{\begin{array}{l}
\frac{\partial \delta_{i}}{\partial s}(s, x)+H_{p}\left(s, x, \phi_{x}\right) \cdot \delta_{i, x}(s, x)=A\left(s, x, \phi_{x}(x)\right) \cdot \delta_{i}(s, x), i=1,2 \\
\delta(0, x)=\left(1_{\mathbb{R}^{d}}, \frac{\partial^{2} \phi^{0}}{\partial y^{0^{2}}}\left(y^{0}\right)\right)^{T}, \text { for } x \in \mathbb{R}^{d}
\end{array}\right.
$$

The unknowns $\delta_{i}$ are $d \times d$ matrix and we here have a matrix equations where the differential operators must be applied component-wise and the $\cdot$ indicates the proper product. The solution of this system, when coupled to (8), gives the necessary information to compute a Eulerian counterpart for $\alpha$ denoted $\beta(s, x)$ :

$$
\beta\left(s, y\left(s, y^{0}\right)\right)=\alpha\left(s, y^{0}\right) .
$$

Finally, $\beta$ can simply be expressed only in terms of Eulerian variables (using (2) (11)):

$$
\beta(s, x)=\left|\delta_{1}(s, x)\right| \text {. }
$$

Alternate methods for the computation of $\beta$ and also the relation between this quantity and wave amplitudes can be found in [20] [9] [32].

\subsection{The Eulerian equations in the $(s, p)$ space}

It is also possible to choose as the Eulerian configuration space, the space spanned by $p\left(s, y^{0}\right)$ instead of $y\left(s, y^{0}\right)$. As long as the $p$ trajectories do not cross, i.e. $\operatorname{det}\left(\frac{\partial p}{\partial y^{0}}\left(s, y^{0}\right) \neq 0\right.$ (see the transversality condition (5)), it is possible to invert $y^{0} \rightarrow p\left(s, y^{0}\right)$ and switch to Eulerian variables now defined on the $(s, p)$ space. As before, we need a variable to describe the phase $\phi(s, p)$ but also one to keep track of the position $y$ which we call $X(s, p)$ :

$$
\begin{aligned}
& X\left(s, p\left(s, y^{0}\right)\right)=y\left(s, y^{0}\right) . \\
& \phi\left(s, p\left(s, y^{0}\right)\right)=\varphi\left(s, y^{0}\right) .
\end{aligned}
$$

ESAIM: Proc., Vol. 11, 2002, 13-40 
Now, simply deriving the above formulae with respect to $s$ and with the help of the Hamiltonian system (1), we can establish that $X$ and $\phi$ satisfy in Eulerian coordinates $(s, p)$ thee equations

(15) $\left\{\begin{array}{l}X_{s}(s, p)-H_{y}(s, X(s, p), p) \cdot X_{p}(s, p)=H_{p}(s, X(s,), p), \\ \phi_{s}(s, p)-H_{y}(s, X(s, p), p) \cdot \phi_{p}(s, p)=p \cdot H_{p}(s, X(s, p), p)-H(s, X(s, p), p) \\ X(0, p)=x, \quad \phi(0, p)=\phi^{0}(x) .\end{array}\right.$

As we will see, the $(s, p)$ space setting is particularly useful when the transversality condition (5) is not satisfied in the $(s, y)$ space. Indeed, It is a classical result in Maslov theory that in this case the equivalent condition cannot degenerate in the $(s, p)$ space. This means that the solution cannot be multi-valued both in the $(s, y)$ and $(s, p)$ configuration spaces.

\section{Comparison of the Lagrangian and viscosity solution in presence of a cuspidal caustic}

The numerical illustrations throughout this paper are performed on a particular Hamiltonian used in two dimensional geometric optics (see [34] or [8]). We assume that the rays possess a privileged direction of propagation which we will choose as the "time" coordinate $s$. The positive smooth index of refraction $n$ therefore may depend both on "time" $s$ and space $x$. The phase necessarily increases in the $s$ direction and the classical two dimensional Eikonal equation can be reduced to and evolution problem of type (8) with $d=1$ and

$$
H(s, x, p)=-\sqrt{n^{2}(s, x)-p^{2}} .
$$

\subsection{The Lagrangian multi-valued solution}

With the choice (16), multi-valuedness may occur either because of the variation $n$ (a lens for instance) or even when $n$ is constant but the initial phase $\phi^{0}$ is such that rays will focus. The simplest case of a cuspidal caustic may be produced by taking :

$$
n \equiv 1, \phi^{0}\left(y^{0}\right)=\int_{0}^{y^{0}} \frac{p^{0}(x)}{\sqrt{1+p^{0}(x)^{2}}} d x, \text { with } p^{0}(x)=\frac{-3(x-0.5)}{\sqrt{1+3(x-0.5)^{2}}},
$$

and may be used to analyze qualitatively the structure of the problem.

Figure 1 shows the corresponding rays, solutions of $(1)$, shot from the bottom $(s=0)$ to the top. The cuspidal caustic can easily be distinguished as the curve which separates the multi-valued zone (where points are reached by three rays) and the single-valued zone (only one ray). In this case it is easy to solve analytically the system (1) and we can represent the level curves of the Lagrangian phase for a continuum of rays. It is done on figure 1. The value of the phase increases from bottom to top. The level curves of the phase fold on themselves inside the caustic where the solution is multi-valued. 

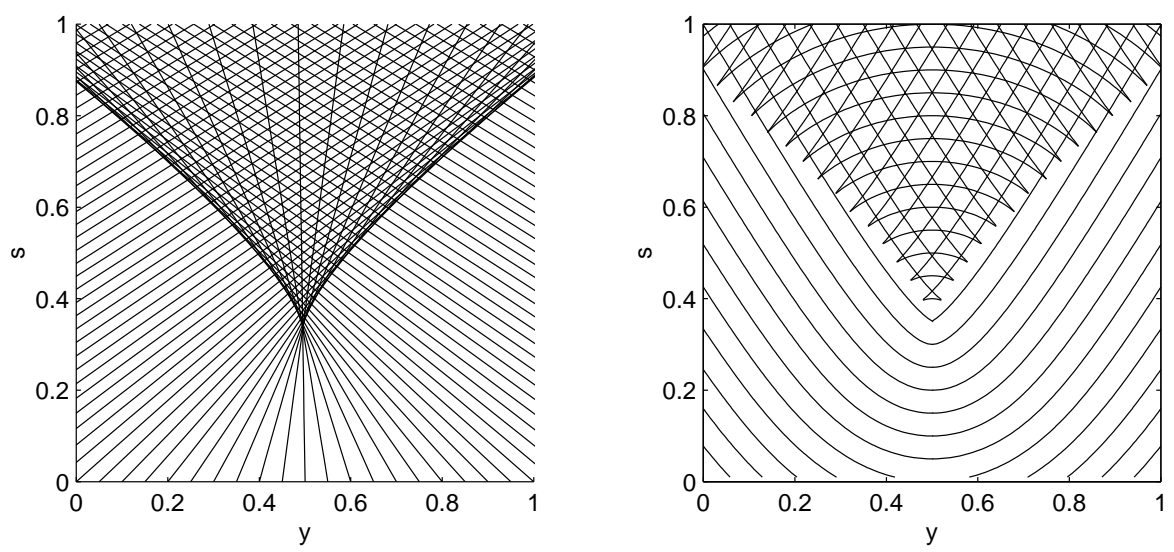

Figure 1: Left: Rays shot from $s=0$. Right: Level curves of $\varphi$, the values of $\varphi$ increase in the $s$ direction.

\subsection{The viscosity single-valued solution}

The viscosity solution is obtained by applying the minimum phase principle (10). The result is displayed on figure 2. It exhibits a curve of singularities in the gradient of $\phi$ called a kink (or shock). The solution can also be obtained by solving the Hamilton-Jacobi equation after discretization and using an upwind scheme. We refer to [28] [33] [1] for the use of such scheme in the Hamilton-Jacobi context.
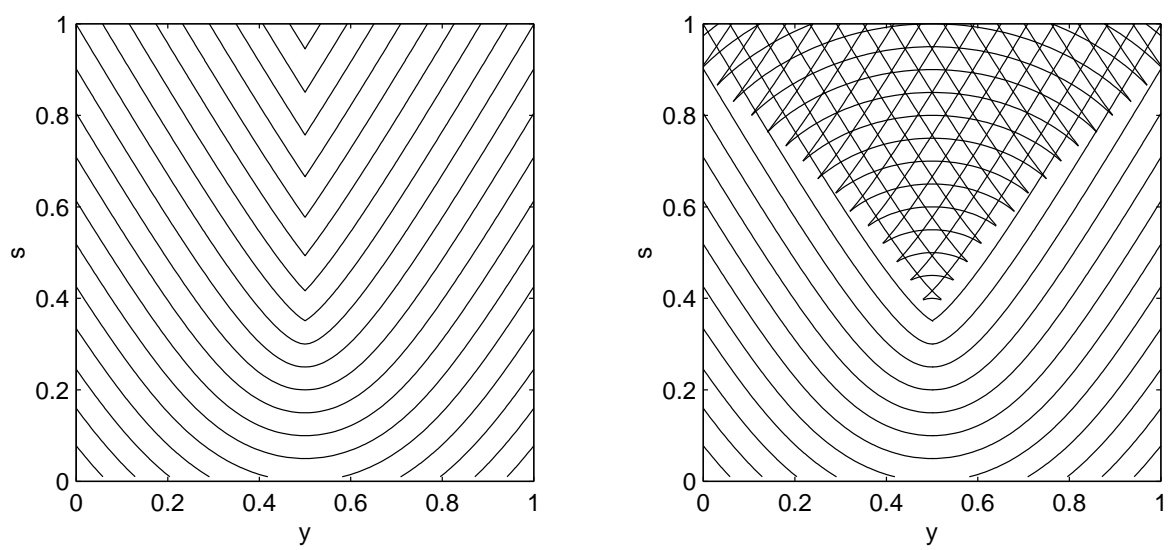

Figure 2: Left : Level curves of the viscosity solution $\phi$ with a kink on $y=0.5$. Right : Level curves of the multi-valued solution $\varphi$. 


\subsection{Branch splitting of the multi-valued solution}

The multi-valued solution can actually be split into three single-valued branches delimited by the caustic curve. On figure 3, we represent the three families of rays or of portions of rays which are associated to each branch and on figure 4 are the corresponding level curves of the phase. The left and right branches $(-\mathrm{Cl}$ and $-\mathrm{Cr})$ are associated to the rays before they reach the caustic, the last branch $(+C)$ is given by the portions of rays once they passed the caustic. A comparison with figure 3 shows that the viscosity solution is composed of the left branch $-C l$ for $y \leq 0.5$ an the right branch $-\mathrm{Cr}$ for $y \leq 0.5$ (they match on the kink $y=0.5$ ). The viscosity solution therefore never "reach" the caustic. By this we mean that the portions of rays implicitly associated to the viscosity solution do not reach the caustic (with the notable exception of the singular tip of the caustic also called the cusp). Superimposing the three plots in each figure gives back respectively the complete family of rays and the multi-valued phase (figure 1).

This a general result valid for any cuspidal caustic, each branch separately can be shown to satisfy an optimal characterization like (9) and is interpreted as the viscosity solution of the Hamilton-Jacobi equation set in the domain corresponding to the actual support of the branch [8]. More precisely, let $\left(s_{c}, x_{c}\right)$, the coordinates of the cusp and $x=C l(s)$ the equation of the right part of the caustic and $x=C r(s)$ of the the left part be given (notice that $x_{c}=C r\left(s_{c}\right)=C l\left(s_{c}\right)$ ). We further assume that the classical solution $\phi^{\text {class }}(s, x)$ is known until "time" $s=s_{c}$. Then, the three branches are viscosity solutions of the original Hamilton-Jacobi equation set in the proper domain with suitable boundary conditions :

(18) $\begin{cases}\phi_{s}^{-C l}(s, x)+H\left(s, x, \phi_{x}^{-C l}(s, x)\right)=0, & \phi^{-C l}\left(s_{c}, x\right)=\phi^{\text {class }}\left(s_{c}, x\right), \quad x<C l\left(s_{c}\right) \\ \phi_{s}^{-C r}(s, x)+H\left(s, x, \phi_{x}^{-C r}(s, x)\right)=0, & \phi^{-C r}\left(s_{c}, x\right)=\phi^{c l a s s}\left(s_{c}, x\right), \quad x>C r\left(s_{c}\right)\end{cases}$

for the $-\mathrm{Cl}$ and $-\mathrm{Cr}$ branches with "out-going" boundary conditions along the caustic (we refer to [8] and for more on the boundary conditions at caustics). And

$$
\phi_{s}^{+C}(s, x)+H\left(s, x, \phi_{x}^{+C}(s, x)\right)=0, \quad\left\{\begin{array}{l}
\phi^{+C}(s, C l(s))=\phi^{-C l}(s, C l(s)) \\
\phi^{+C}(s, C r(s))=\phi^{-C r}(s, C r(s))
\end{array}\right.
$$

for the last branch $+C$ which is connected to the $-C$ branches via the initial conditions. For each branch separately, the phase is nowhere discontinuous and except at the caustic its gradient is well defined. We have $\phi_{x}\left(s, y\left(s, y^{0}\right)\right)=p\left(s, y^{0}\right)$ for the corresponding Eulerian and Lagrangian branches.

\section{The numerical algorithm}

This section explains how the branch splitting algorithm of section 4.3 can be used to provide a tractable solution for a general cuspidal caustic. The resolution of equations (19) and (18) is subject in particular to the numerical localization of caustics which are used to define the support 

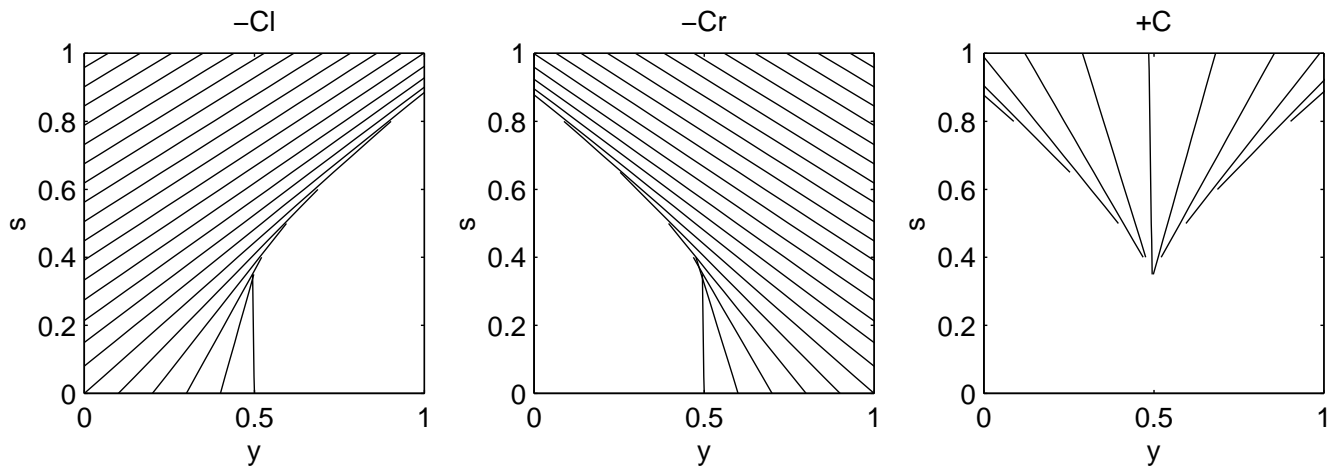

Figure 3: From left to right : the families of ray associated to the three different branches of the Lagrangian solution. The caustic is used to split the rays. In particular the branch $+C$ is made of the remaining part of the rays of the branches $-C$ s stopped at the caustic.
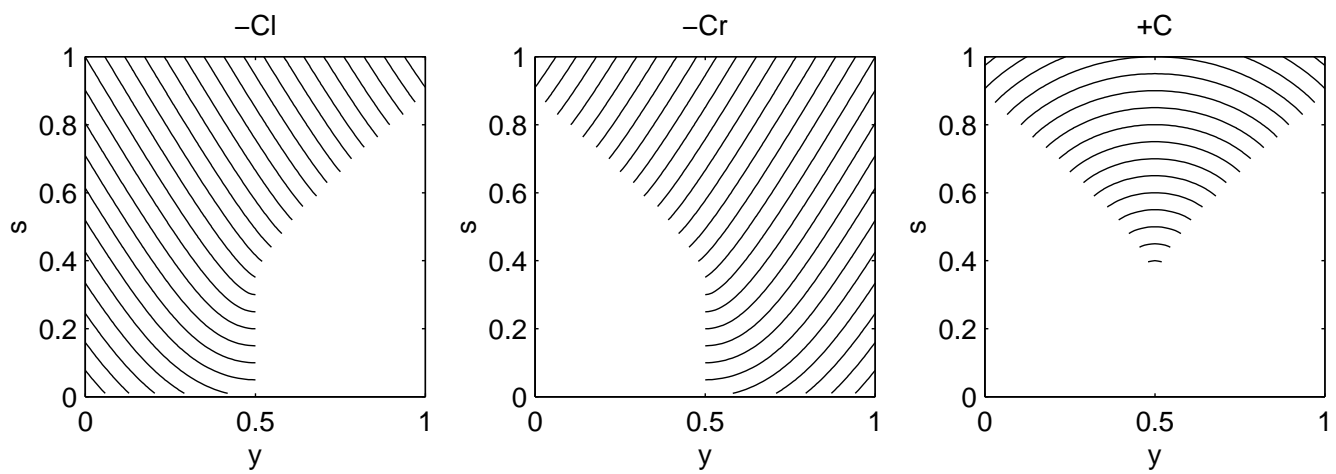

Figure 4: From left to right: The corresponding level curves of the phase. The values of the branches $-C$ s and $+C$ match on their respective part of the caustic. 
of each branch. Eulerian caustic localization is itself a delicate problem for which we already proposed a "time asymptotic method" [10]. We present here a different and somewhat simpler method which combines the resolution of an ODE for the caustic curve with an adaptative gridding of the Lagrangian domain.

The program of our algorithm can be briefly summarized as follow : The "time asymptotic" method is modified to compute precisely the position if the tip of the cusp $\left(s_{c}, x_{c}\right)$ and the classical solution $\phi^{\text {class }}\left(s_{c},.\right)$. We then switch to $(s, p)$ space locally around $\left(s_{c}, x_{c}\right)$ to initialize the branch splitting algorithm. In this configuration and locally the solution is single-valued but may not remain so. We therefore go back to $(s, y)$ space to realize the branch splitting algorithm.

The method is illustrated using the example of section 4 and a more complicated test case is presented in section 6 .

\subsection{Computing the classical solution}

An accurate resolution of the multi-valued problem cannot afford a loose approximation of the caustic geometry on a Cartesian space-time grid discretization. We propose here a simplification of the method presented in [10] to determine accurately the time $s_{c}$ of occurrence of the cusp as well as the classical solution at this horizon.

We recall that the Eulerian geometrical spreading, defined as

$$
\beta\left(s, y\left(s, y^{0}\right)\right)=\operatorname{det}\left(\frac{\partial y}{\partial y^{0}}\left(s, y^{0}\right)\right)=\operatorname{det}\left(\delta_{1}\left(s, y\left(s, y^{0}\right)\right)\right)
$$

can be computed using an Eulerian system of transport equations (12). On each time slice $s$ we define

$$
\tilde{\beta}(s)=\min _{x} \beta(s, x) .
$$

And introduce a new time variable as

$$
\tilde{s}(s)=\int_{0}^{s} \frac{1}{\tilde{\beta}(\tau)^{r}} d \tau
$$

where the exponent $r$ is a degree of freedom of the method.

We remember now that geometrical spreading goes to 0 as rays approach the caustic. This will also be the behavior of the Eulerian $\beta(s, x)$ and consequently of $\tilde{\beta}(s)$

$$
\tilde{\beta}(s) \rightarrow 0 \text { when } s \rightarrow s_{c} .
$$

If $r$ is properly chosen we can expect that $\tilde{s}(s)$ will behave like $\log \left(\frac{1}{s_{c}-s}\right)$ near $s_{c}$. Even though $r$ can be determined analytically at the continuous level, the discretization of equation (12) will ruin the exact convergence property (20) and some dependence of $r$ on the discretization should be studied. For now we just adjust heuristically $r$ to produce the correct convergence and the expected behavior. A rigorous study of this point has yet to be done. 
The next step is to perform the change of time variable in the unknowns of our problem

$$
\{\tilde{\phi}(\tilde{s}, x), \tilde{\delta}(\tilde{s}, x), S(\tilde{s})\}=\{\phi(s, x), \delta(s, x), s\}
$$

augmented with the unknown $S$ which simply keeps track of the old time variable $s$ and which will be use to invert this change of variable in time. As $\tilde{s}$ goes to $+\infty$ (as a $\log )$ the new $\tilde{\text {. variables }}$ behave (exponentially fast) like the old ones as $s$ goes to $s_{c}$, the first caustic time.

The equations in the $(\tilde{s}, x)$ coordinates are (simply apply $\tilde{\beta} d \tilde{s}=d s$ to (8) and (12))

$$
\left\{\begin{array}{l}
\tilde{\phi}_{\tilde{s}}(\tilde{s}, x)+\tilde{\beta}(\tilde{s}) H\left(s, x, \tilde{\phi}_{x}(\tilde{s}, x)\right)=0, \quad \tilde{\phi}(0, x)=\phi^{0}(x) \\
\left.\tilde{\delta}_{i, \tilde{s}}(\tilde{s}, x)+\tilde{\beta}(\tilde{s})\left(H_{p}\left(\tilde{s}, x, \tilde{\phi}_{x}\right) \cdot \tilde{\delta}_{i, x}(\tilde{s}, x)-A\left(\tilde{s}, x, \tilde{\phi}_{x}(x)\right) \cdot \tilde{\delta_{i}} \tilde{(} \tilde{s}, x\right)\right),+c . i . \\
S_{\tilde{s}}(\tilde{s})=\tilde{\beta}(\tilde{s}), \tilde{S}(0)=0
\end{array}\right.
$$

In practice we wait for these evolution equations to be stationary and we then invert the change of variable to recover the classical solution, i.e the solution of the Hamilton-Jacobi equation until $s=s_{c}$ (see figure 5):

$$
\phi^{c l a s s}(S(\tilde{s}), x)=\tilde{\phi}(\tilde{s}, x) .
$$
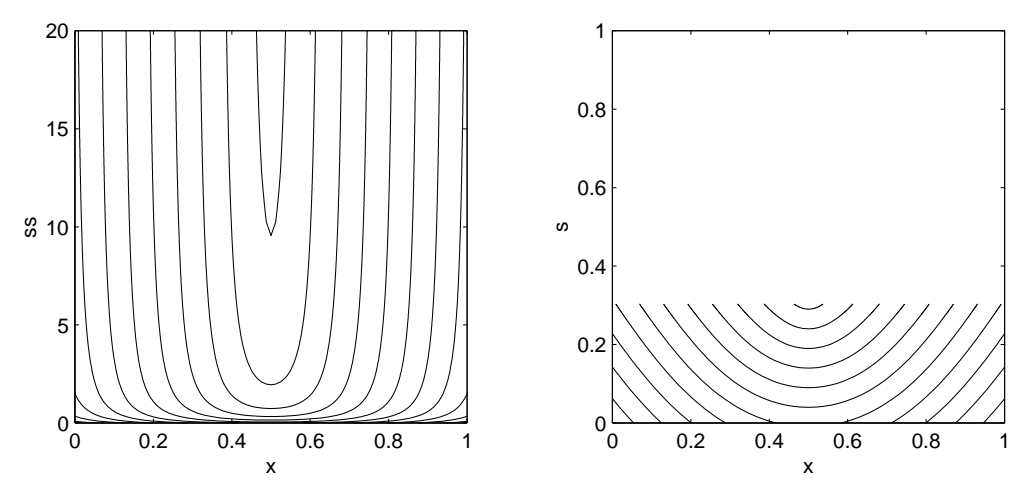

Figure 5: Left : Level curves of $\tilde{\phi}(\tilde{s}, x)$. Right : Level curves of $\phi^{\text {class }}(S, x)$. In this case $s_{c}=0.3032$.

\subsection{Localization of $x_{c}$ in $(s, p)$ space}

Section 5.1 explains how $s_{c}$ can be accurately determined numerically. We now discuss the localization of $x_{c}$. It is clear that the minimum of $\beta\left(s_{c}, x\right)$ is achieved at $x_{c}$. However, because we work on a finite difference grid, we can only hope to be accurate up to the space discretization. This is not sufficient for our purpose of capturing accurately the multi-valued solution which is "branching" at $\left(s_{c}, x_{c}\right)$. One possibility is to use adaptative gridding in space (in the spirit of [32] for instance or by using $\beta$ as a local grid refinement criterium). This solution may however be slow 
because the CFL condition will decrease dramatically as $\tilde{\beta}$ goes to 0 . We propose here another approach, more natural in a sense, which will also be used to initialize the multi-valued solution.

We know that the Lagrangian sub-manifold $\Lambda$ (section 2.2) can be parameterized using $(s, p)$ as a polynomial of degree 3 in $p$, hence the 3 branches developing inside the caustic correspond to each of the three roots in $p$. At $s=s_{c}$, the solution is however not yet multi-valued, we therefore assume that $\Lambda$ can be approximated locally by a polynomial of degree 3 which has a single real root. Using the notations of section 3.4 , we write in $(s, p)$ space

$$
X\left(s_{c}, p\right)=x_{c}+a\left(p-p_{c}\right)^{3}
$$

where $\left(s_{c}, x_{c}, p_{c}\right)$ are the coordinates of the tip of the cusp in phase space ; $x_{c}$ and $p_{c}$ are not known and $a$ is a constant coefficient also unknown. A similar polynomial representation for the phase $\phi$ is also available which is necessarily a polynomial of degree 4 in $p$ (see the references in section 2.2)

$$
\phi\left(s_{c}, p\right)=\phi_{c}+3 a\left(\frac{\left(p-p_{c}\right)^{4}}{4}+p_{c} \frac{\left(p-p_{c}\right)^{3}}{3}\right)
$$

The coefficients are obtained by consistency with (21) and using the fact that except at $X=x_{c}$, we must have $p=\phi_{X}$. Remark that $\phi_{c}$ the phase at $\left(s_{c}, x_{c}, p_{c}\right)$ is also unknown.

We now want to determine the unknown parameters $\left(a, x_{c}, p_{c}, \phi_{c}\right)$. Let $x^{-}$and $x^{+}$be two neighboring points of $x_{c}$. At these points, the phases $\phi^{\text {class }}\left(s_{c}, x^{-}\right)$and $\phi^{\text {class }}\left(s_{c}, x^{+}\right)$and its gradient are a priori known. Furthermore, setting $p^{-}=\phi_{x}^{\text {class }}\left(s, x^{-}\right)$and $p^{+}=\phi_{x}^{\text {class }}\left(s, x^{+}\right)$we have

$$
\left\{\begin{array}{l}
x^{-}=X\left(s_{c}, p^{-}\right), x^{+}=X\left(s_{c}, p^{+}\right), \\
\phi^{\text {class }}\left(s_{c}, x^{-}\right)=\phi\left(s_{c}, p^{-}\right), \phi^{\text {class }}\left(s_{c}, x^{+}\right)=\phi\left(s_{c}, p^{+}\right) .
\end{array}\right.
$$

In practice, $x^{-}$and $x^{+}$are discretization points, neighbors to $x_{c}$, and $p^{-}, p^{+}, \phi^{\text {class }}\left(s_{c}, x^{-}\right)$and $\phi^{\text {class }}\left(s_{c}, x^{+}\right)$are outputs of the numerical method described in section 5.1. With the help of (21) (22), we transform (23) in the system

$$
\left\{\begin{array}{l}
x^{-}=x_{c}+a\left(p^{-}-p_{c}\right)^{3} \\
x^{+}=x_{c}+a\left(p^{+}-p_{c}\right)^{3} \\
\phi^{\text {class }}\left(s_{c}, x^{-}\right)=\phi_{c}+3 a\left(\frac{\left(p^{-}-p_{c}\right)^{4}}{4}+p_{c} \frac{\left(p^{-}-p_{c}\right)^{3}}{3}\right. \\
\phi^{\text {class }}\left(s_{c}, x^{+}\right)=\phi_{c}+3 a\left(\frac{\left(p^{+}-p_{c}\right)^{4}}{4}+p_{c} \frac{\left(p^{+}-p_{c}\right)^{3}}{3}\right.
\end{array}\right.
$$

where $\left(a, x_{c}, p_{c}, \phi_{c}\right)$ are the unknown. System (24) can be solved analytically or using an iterative method. This is of course one of the many ways this parameter identification can be performed, but it seems to work and we did not experimented other possibilities.

The result is illustrated on figure 6 and 7. For our symmetrical example $x_{c}$ is right at a discretization point. A less trivial situation is treated in section 6 . 

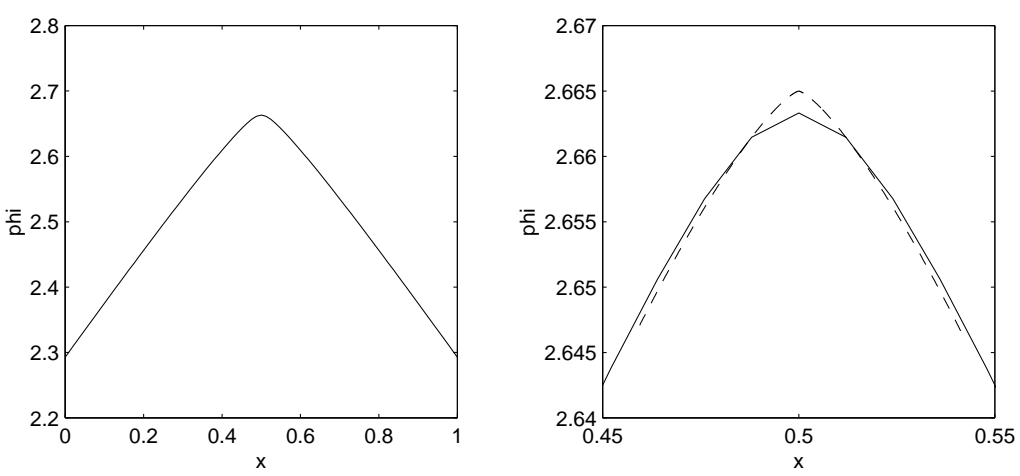

Figure 6: Left : phase at $s=s_{c}$ as a function of $x$. Right : a zoom near the cusp, the broken lines indicates the discretization. The dashed line is the graph of $\phi$ versus $X$ based on the numerical extrapolations (21-22).
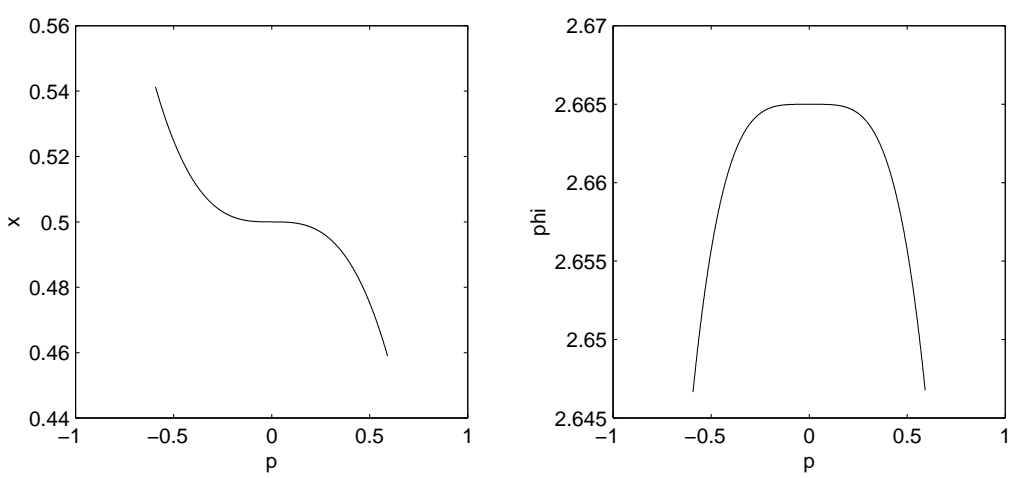

Figure 7: $X$ and $\phi$ as a function of $p$ at $s=s_{c}$ based on the numerical extrapolations (21-22). 


\subsection{Initialization of the three branches}

Locally around $\left(s_{c}, x_{c}\right)$, we know that we can safely work in the $(s, p)$ space configuration. This means that $X$ and $\phi$ will remain well defined smooth single valued functions of $(s, p)$. Note that this need not remain so for all times and therefore we should keep in mind that we need to go back rather quickly (to avoid caustics in the $(s, p)$ space) to the $(s, y)$ configuration space and perform the splitting of the anticipated caustic according to section 4.3. From $s=s_{c}$, we therefore proceed to solve equations (15). We use finite differences on a local domain centered around $p_{c}$. The initialization is given by (21-22) and we implemented out-going boundary conditions. This is not necessarily correct but does not pollute the interior of our $p$ domain. Figure 8 shows the result at $s=s_{c}$. At each end of the solution, one can observe the two Folds of the Lagrangian sub-manifold. The caustics correspond to the points where $X_{p}=0$.
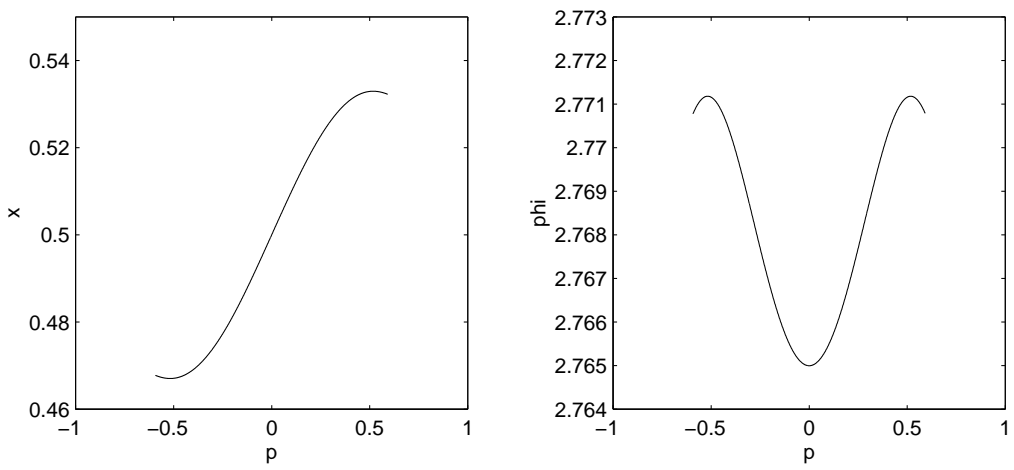

Figure 8: $X$ and $\phi$ as a function of $p$ at $s=s_{c}+0.1$.

Away from $x_{c}$ where there is no multi-valuedness we can remain in the $(s, y)$ configuration and concurrently solve the Hamilton-Jacobi equation (8). It is then possible to reconstruct each branch of the multi-valued solution by "gluing" this $(s, y)$ computation and the $(s, p)$ computation switched back to $(s, y)$ space. This is simply done by representing $\phi(s, p)$ as a function of $X(s, p)$. We show the multi-valued solution at $s=s_{c}+0.03$ and $s=s_{c}+0.1$ on figures 9 and 10 . We insist here that caution should be exercised in the return to $(s, y)$ process. An interpolation procedure is involved which depends on the size of the discretization. In particular, it is absolutely necessary to let the $(s, p)$ computation evolve long enough for the $X$-span of the $+C$ branch to cover at least several mesh sizes in $x$.

In this section, we initialized the three branches by "zooming" on the the cusp in the $(s, p)$ space. Now that each branch is not reduced to a point in space and has a real numerical existence on a finite difference grid in the $(s, y)$ space configuration (see figure 10), we possess a suitable initialization for our splitting algorithm (18-19). The resolution of this coupled system relies on two additional ingredients (next two sections). The addition of two ODEs for the computation of the caustics curves (Folds) and some change of variables which "straighten" the caustics and makes it possible to work on regular Cartesian grid. 

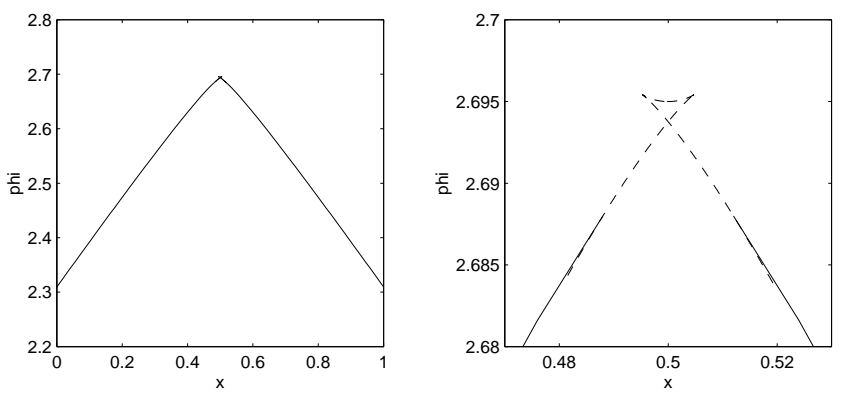

Figure 9: $\phi$ at $s=s_{c}+0.03$ (zoom on the right), solid line $(s, y)$ computation, dashed line $(s, p)$ computation.
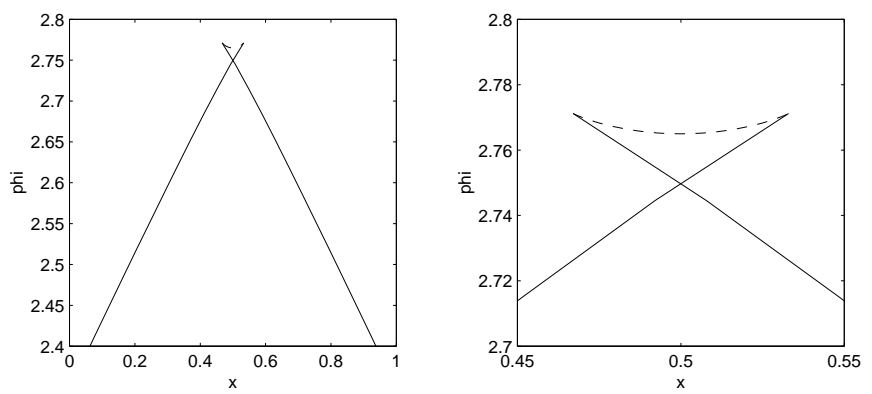

Figure 10: $\phi$ at $s=s_{c}+0.1$ (zoom on the right), solid line : $-C l$ and $-C r$ branches, dashed line $+C$ branch. 


\subsection{Localizing the Folds}

The two Folds $C r$ and $C l$ are treated likewise. We therefore drop the the left-right dependence and explain how to derive an ordinary differential equation for $C(s) ; x=C(s)$ being the equation of a Fold caustic.

Let us first introduce $\left\{\left(s, C(s), p_{c}(s)\right), s>s_{c}\right\}$ the trajectory of the caustic in the phase space $\mathbb{R}_{s}^{+} \times \mathbb{R}_{y} \times \mathbb{R}_{p}$. This curve belongs in particular to $\Lambda$ but is not a bicharacteristics.

We recall (section 4.3) that except at the caustic we can identify $p$ to the gradient of the phase (of one of the branches) : $\phi_{x}\left(s, y\left(s, y^{0}\right)\right)=p\left(s, y^{0}\right)$. Like for the initialization of the cusp, the good setting at the caustic is the $(s, p)$ space configuration and we have (again with the notations of section 3.4)

$$
\left\{\begin{array}{l}
C(s)=X\left(s, p_{c}(s)\right)=y\left(s, y_{c(s)}^{0}\right) \\
p_{c}(s)=p\left(s, y_{c(s)}^{0}\right) \\
X_{p}\left(s, p_{c}(s)\right)=0
\end{array}\right.
$$

where $y_{c(s)}^{0}$ is the initial position of the bicharacteristics which reaches the $\left(s, C(s), p_{c}(s)\right)$ phase space point. The second equation simply says that the Lagrangian sub-manifold folds at $(s, C(s))$ in the $p$ dimension direction. Using (25) and remembering that, at the caustic $\frac{\partial y\left(s, y^{0}\right)}{\partial y^{0}}=0$ (we are in 1-D), we find

$$
\begin{aligned}
\dot{C}(s) & =\dot{y}\left(s, y_{C(s)}^{0}\right) \\
& =H_{p}\left(C(s), p_{c}(s)\right) .
\end{aligned}
$$

This is not surprising as we know the caustic to be the envelope of the rays. The essential difference with the first equation of the Hamiltonian system (1) is the following : at a caustic point $(s, C(s))$ we need to know $p_{c}(s)$ which is nothing but the $p$ component $p\left(s, y_{c(s)}^{0}\right)$ of the bicharacteristics issued from $y_{c(s)}^{0}$ (also unknown). As already mentioned, $p_{c}(s)$ cannot be characterized directly using the phase. We here derive an approximate characterization of $p_{c}(s)$ depending on the gradient of the phases $\phi^{-C}(s,$.$) and \phi^{+C}(s,$.$) which are connected at the caustic point (s, C(s))$. It gives the necessary closure for a coupled system formed by (18-19) and two equations of type (26) for $C r(s)$ and $C l(s)$.

We work with fixed $s$ and remember that the Lagrangian sub-manifold $\Lambda$ (section 2.2) can be parameterized using $(s, p)$ as a polynomial of degree 2 in $p$. We therefore expect that a good approximation of $X(s, p)$ locally around $\left(s, p_{c}(s)\right)$ is given by

$$
X(s, p)=C(s)+a\left(\left(p-p_{c}(s)\right)^{2}\right.
$$

where $\left(a, p_{c}(s)\right)$ are unknown. There are no term of degree 1 because $X_{p}(s, p)$ satisfies $X_{p}\left(s, p_{c}(s)\right)=$ 0 . Let $\left(x^{-}, x^{+}\right)$be neighboring points of $C(s)$ on the support of the $-C$ and $+C$ branches. We denote $p^{-}=\phi_{x}^{-C}\left(s, x^{-}\right)$and $p^{+}=\phi_{x}^{+C}\left(s, x^{+}\right)$. According to (27), $\left(a, p_{c}(s)\right)$ is the solution of the system

$$
\left\{\begin{array}{l}
x^{-}=C(s)+a\left(\left(p^{-}-p_{c}(s)\right)^{2}\right. \\
x^{+}=C(s)+a\left(\left(p^{+}-p_{c}(s)\right)^{2}\right.
\end{array}\right.
$$

ESAIM: Proc., Vol. 11, 2002, 13-40 
which can be solved analytically or using a Newton method. In the simplest case (which is not our case, see next section) where $C(s)-x^{-}=C(s)-x^{+}$, we simply find $p_{c}(s)=0.5\left(\phi_{x}^{-C}\left(s, x^{-}\right)+\right.$ $\left.\phi_{x}^{+C}\left(s, x^{+}\right)\right)$, i.e. the direction of the caustic is approximated by the mean of the direction of nearby rays at a crossing point.

In practice, $x^{-}$and $x^{+}$are chosen as discretization points for respectively the $-C$ and $+C$ branches where the gradient of the phase is approximated. Regarding time discretization, we only say that both implicit and explicit schemes have been experimented on the full coupled system. Implicit schemes do not seem to work better.

\subsection{Straightening caustics}

In this section we explain how the computational domain can be "straightened" to a regular square Cartesian grid using a dynamic change of variable in space.

We first address the case of the $-C$ branches. They are bounded by the caustic on one side and we choose to close the domain on the other side with out-going boundary conditions which may be inconsistent with the problem but do not interfere with the solution away from this "artificial" boundary. Let us introduce the following change of coordinates

$$
(s, \tilde{x}(s, x)+C(s)) \leftarrow(s, x),
$$

which simply shifts each time slice such that the domain is bounded on the caustic side by $\tilde{x}=0$. We introduce, like in section 5.1, a new phase variable depending on $(s, \tilde{x})$

$$
\tilde{\phi}(s, \tilde{x})=\phi^{-C}(s, x),
$$

and establish (with the help of (26)) that it satisfies the modified equation

$$
\tilde{\phi}_{s}(s, \tilde{x})+H\left(s, \tilde{x}+C(s), \tilde{\phi}_{\tilde{x}}(s, \tilde{x})\right)-H_{p}\left(s, C(s), p_{c}(s)\right) \cdot \tilde{\phi}_{\tilde{x}}(s, \tilde{x})=0
$$

with out-going boundary condition at $\tilde{x}=-M, \mathrm{M}$ is given by the user. At the caustic $(\tilde{x}=0)$, we can either use out-going boundary conditions as the scheme obviously needs to upwind from the interior of the domain or impose the consistent boundary condition $\tilde{\phi}_{\tilde{x}}(s, 0)=p_{c}(s)$. Equation (30) is still an Hamilton-Jacobi equation with convex Hamiltonian. Some care is needed to define the numerical Hamiltonian but otherwise the same upwind scheme can be used.

We recover the solution in $(s, x)$ by inverting the change of coordinates (29):

$$
\phi(s, \tilde{x}+C(s))=\tilde{\phi}^{-C}(s, \tilde{x})
$$

The result for the $-C$ branches are displayed on figure 11 and 12. The method integrates both caustics localization (and therefore also depends on the resolution of the $+C$ branch) and this change of variable technique. 

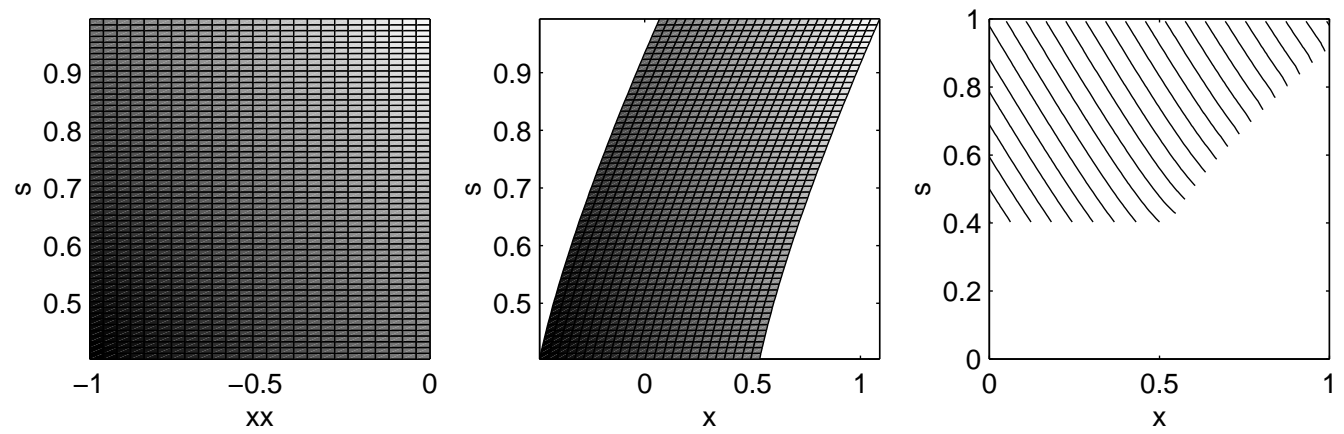

Figure 11: $-C l$ branch. Left : gray-scale plot of $\phi^{-C l}$ as a function of $(s, \tilde{x})$. Center : gray-scale plot of $\phi^{-C l}$ as a function of $(s, x)$, we recover the shape of the caustic. Right : Level curves of $\phi^{-C l}$ as a function of $(s, x)$.
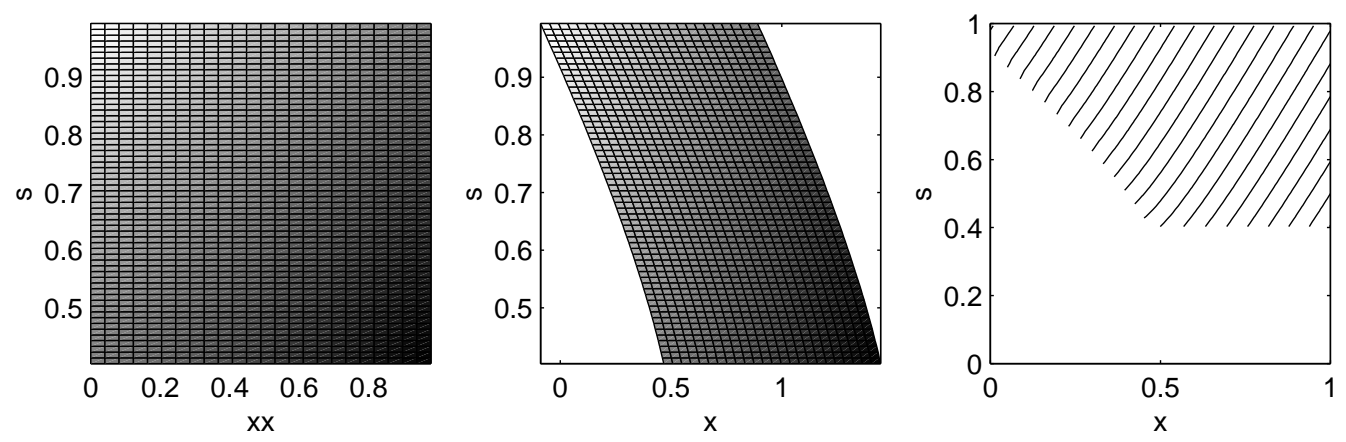

Figure 12: $-\mathrm{Cr}$ branch. As figure 11. 
The case of the $+C$ branch is a bit more complicated as it is bounded on both side by a caustic curve solution of (26). The proposed solution in this case is to make a linear change of coordinates

$$
(s, a(s) \tilde{x}(s, x)+b(s)) \leftarrow(s, x)
$$

where $a$ and $b$ are dynamically defined by (the left and right dependence are made explicit on the caustic curve)

$$
\left\{\begin{array}{l}
a(s)=\frac{C l(s)-C r(s)}{2 M} \\
b(s)=\frac{C l(s)+C r(s)}{2}
\end{array}\right.
$$

$\tilde{x}$ here spans the interval $]-M, M[$ here $M$ is fixed by the user. We again perform a change of variable

$$
\tilde{\phi}(s, \tilde{x})=\phi^{+C}(s, x)
$$

and the new variable satisfies

$$
\tilde{\phi}_{s}(s, \tilde{x})+H\left(s, a(s) \tilde{x}+b(s), \frac{\tilde{\phi}_{\tilde{x}}(s, \tilde{x})}{a(s)}\right)-(\dot{a}(s) \tilde{x}+\dot{b}(s)) \cdot \frac{\tilde{\phi}_{\tilde{x}}(s, \tilde{x})}{a(s)}=0
$$

for $\tilde{x} \in]-M, M\left[\right.$. The boundary conditions are given by (19) : $\phi^{+C}(s, C l(s))=\phi^{-C l}(s, C l(s)$ and $\phi^{+C}(s, C r(s))=\phi^{-C r}(s, C r(s))$.

This equation is also tractable using an upwind scheme. We invert the linear change of coordinate and recover our $+C$ branch in the standard coordinates system $(s, x)$ (figure 13).
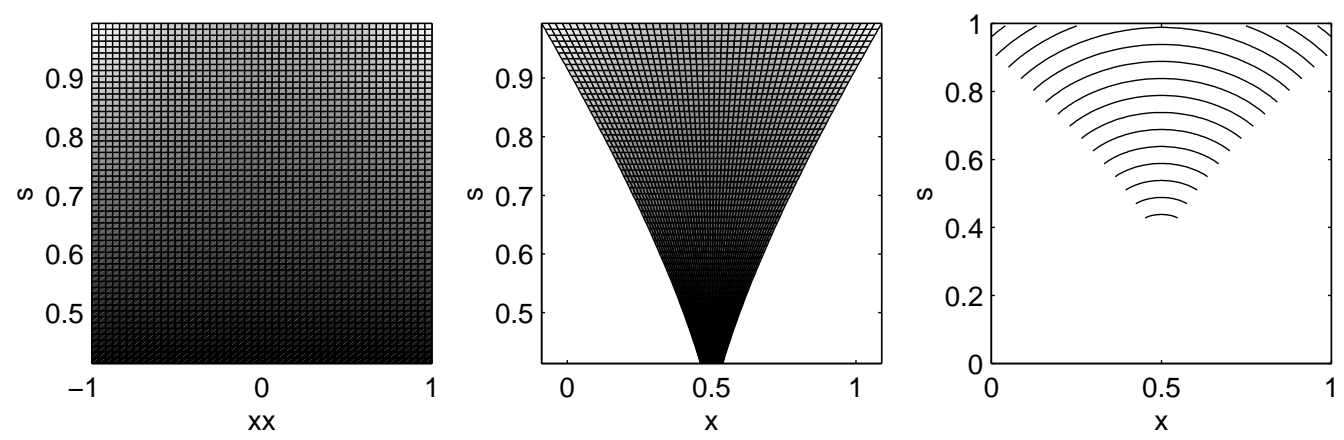

Figure 13: $+C$ branch. As figure 11 .

\subsection{Multi-valued solution}

The only remaining processing to be done is to superimpose the three branches and the classical solution on the same plot. This is figure 14. The white band with no solution correspond to the 


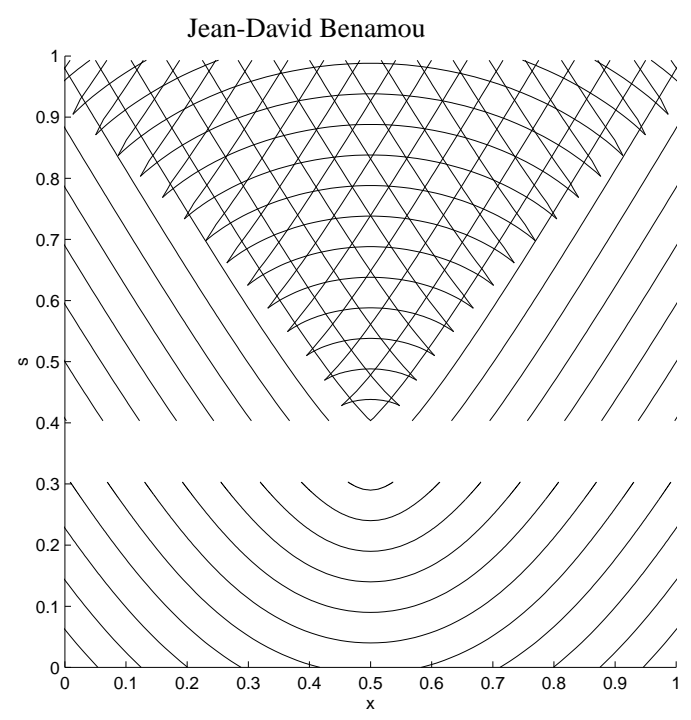

Figure 14: The Eulerian multi-valued solution.

time window of the $(s, p)$ treatment of the cusp. A more thorough check is to compare the phases computed at time $s=1$ using a Lagrangian (ray) method and our method. It is done on figure 15 .

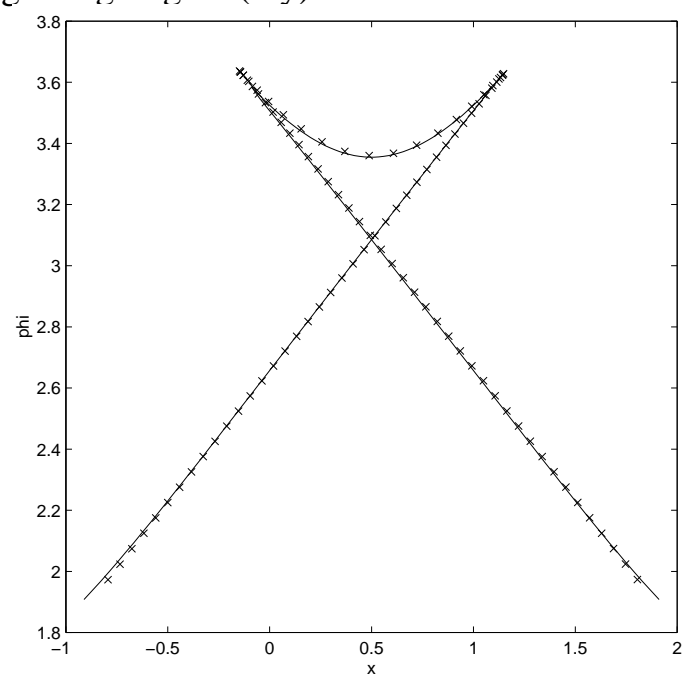

Figure 15: Phase at $s=1$. Solid line : Eulerian. Crosses : Lagrangian.

\section{Numerical test}

We test our Eulerian method on a more complicated and non symmetric cuspidal caustic. The Hamiltonian is still given by (16) but the index of refraction defines a waveguide (figure 16) $n(y)=\frac{1}{1+0.2 \sin (3 \pi y)}$. The initial phase $\phi^{0}\left(y^{0}\right)=\int_{0}^{y^{0}} \frac{n\left(y^{0}\right)\left(y^{0}-0.3\right)}{\sqrt{1+\left(y^{0}-0.3\right)^{2}}} d x$ is non symmetric with respect to $n$. 


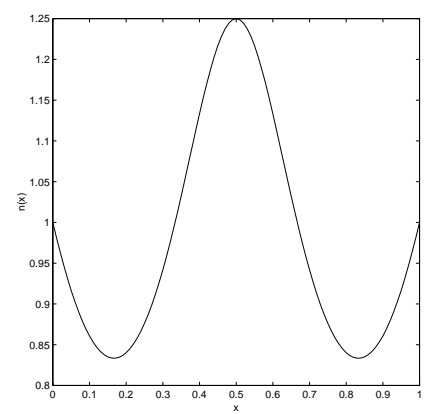

Figure 16: Index $n(y)$.

The figures 17 to 25 illustrate the numerical treatments detailed in section 5. Figure captions are explicit.
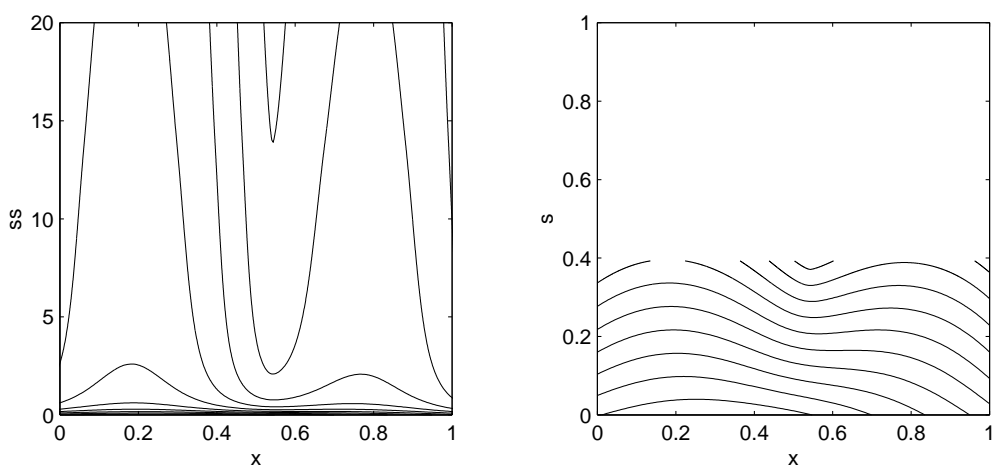

Figure 17: Left : Level curves of $\tilde{\phi}(\tilde{s}, x)$. Right : Level curves of $\phi^{\text {class }}(S, x)$. In this case $s_{c}=0.3927$.

\section{Conclusion}

Figures 24 and 25 illustrates the ability of the method to compute an arbitrary cuspidal caustic. It is difficult to compare execution times between the Eulerian and the Lagrangian method because the formats of the results are different. The Eulerian method was however faster in producing the phase profile (figure 21) because a large number of rays were needed to recover a few points on the $-C$ branches and the Eulerian $x$ discretization was rather coarse (40 points). The "Lagrangian" changes of variable of section 5.5 has the nice feature of automatically refining the grid where needed (where the ray density is high) and hence improving the accuracy (we used first order schemes). 

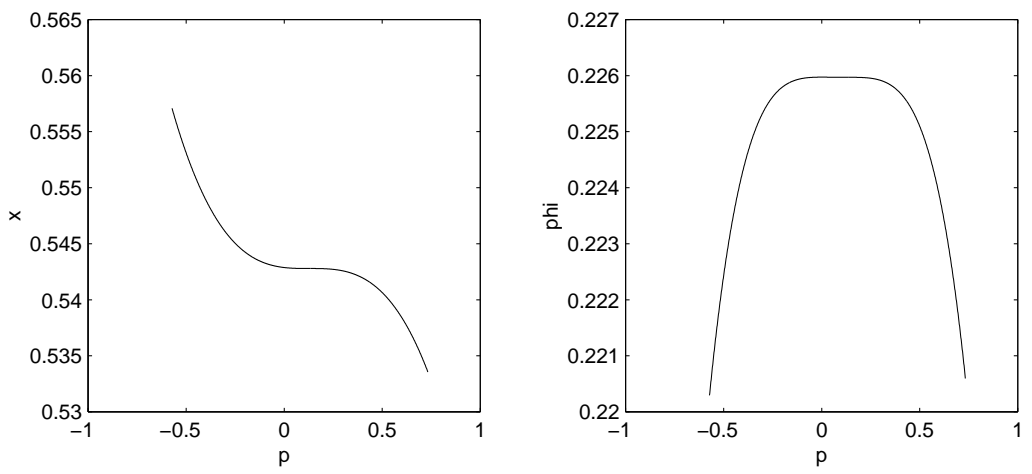

Figure 18: $X$ and $\phi$ as a function of $p$ at $s=s_{c}$ based on the numerical extrapolations (21-22).
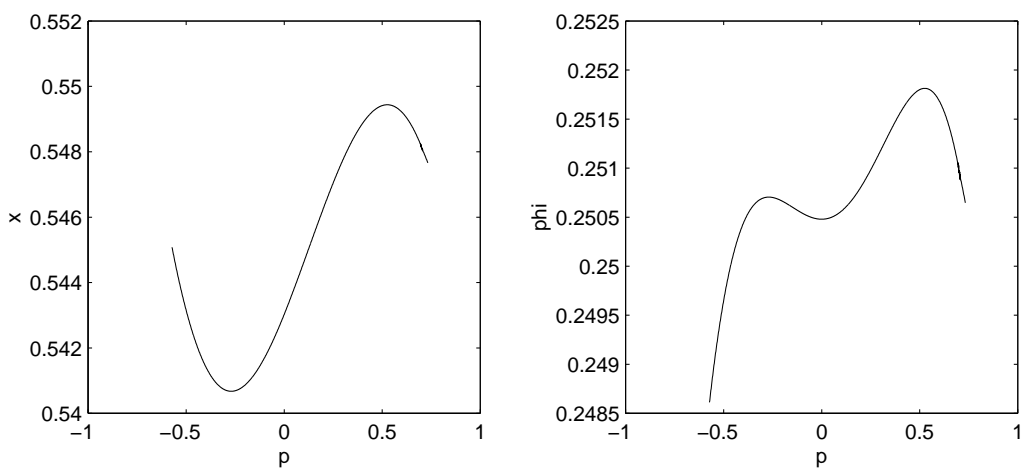

Figure 19: $X$ and $\phi$ as a function of $p$ at $s=s_{c}+0.02$.
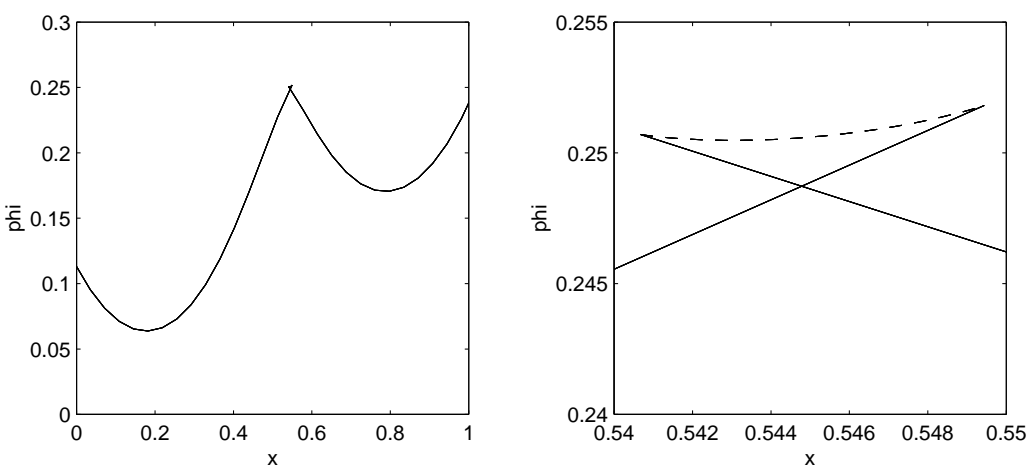

Figure 20: $\phi$ at $s=s c+0.02$ (zoom on the right), solid line : $-C l$ and $-C r$ branches, dashed line $+C$ branch. 

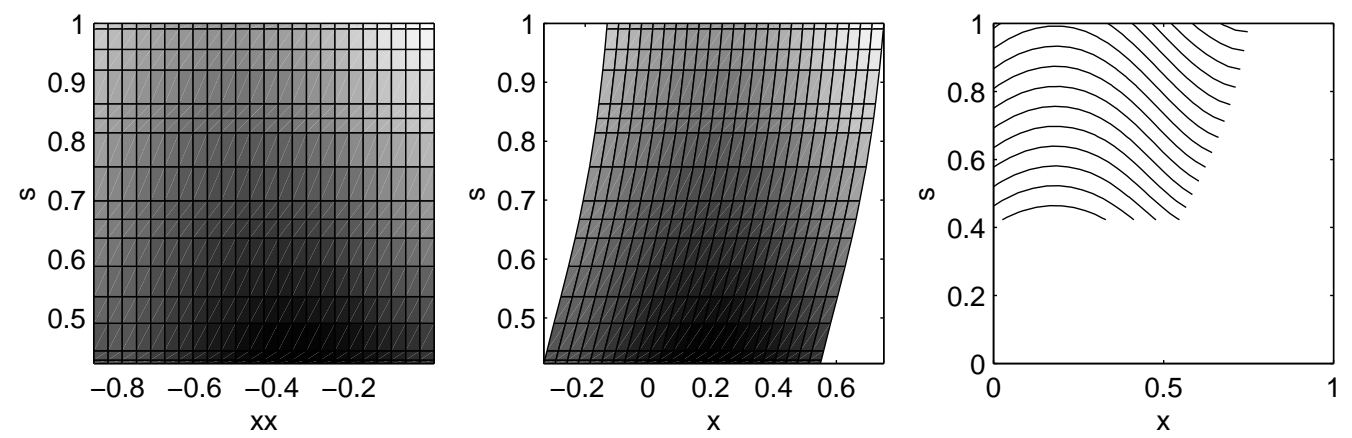

Figure 21: $-C l$ branch. Left : gray-scale plot of $\phi^{-C l}$ as a function of $(s, \tilde{x})$. Center : gray-scale plot of $\phi^{-C l}$ as a function of $(s, x)$, we recover the shape of the caustic. Right : Level curves of $\phi^{-C l}$ as a function of $(s, x)$.
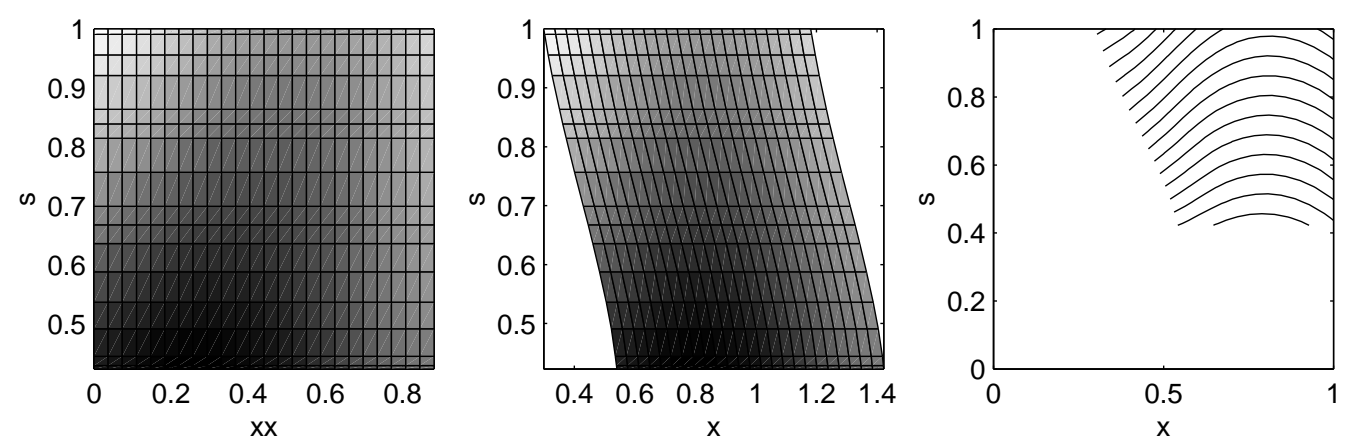

Figure 22: $-C r$ branch. As figure 21.
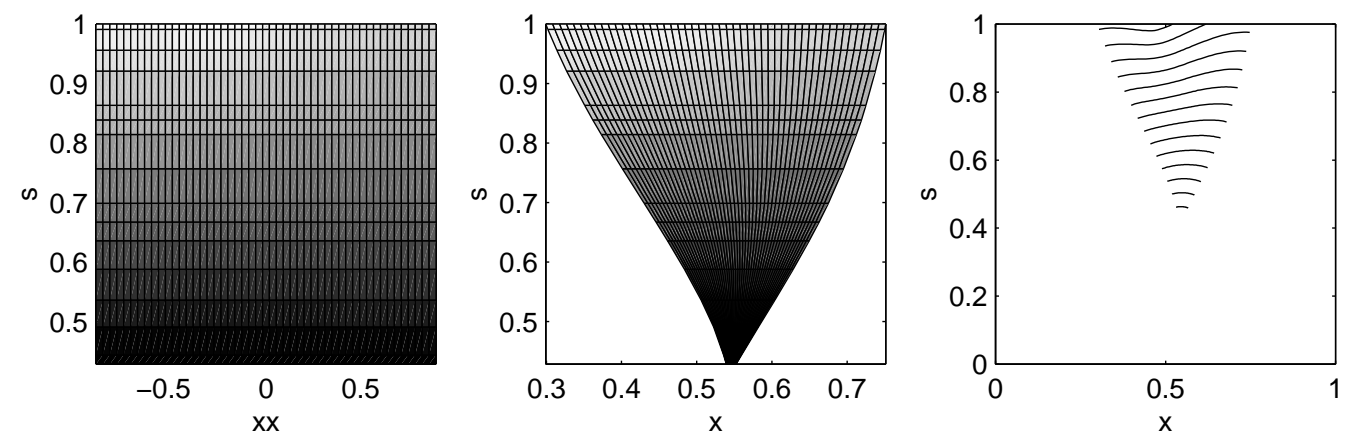

Figure 23: $+C$ branch. As figure 21 . 

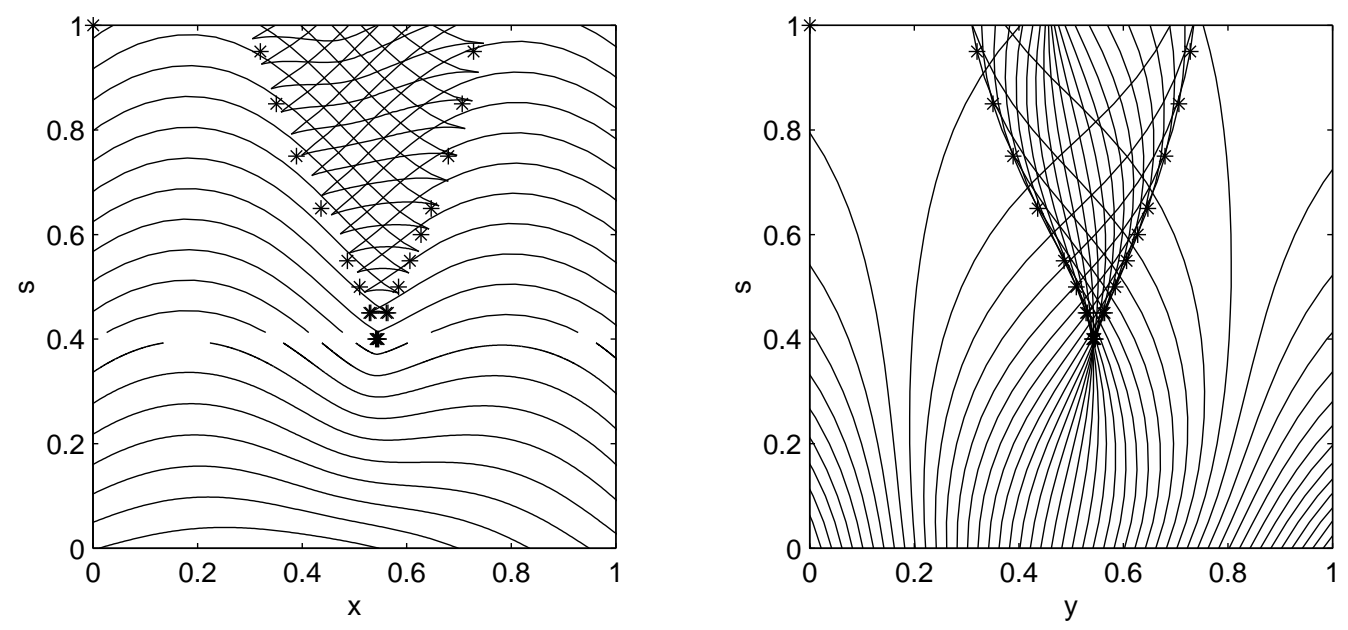

Figure 24: Left : Eulerian multi-valued solution . Right : Lagrangian solution (rays). The Lagrangian caustic points are represented by stars on both plots.

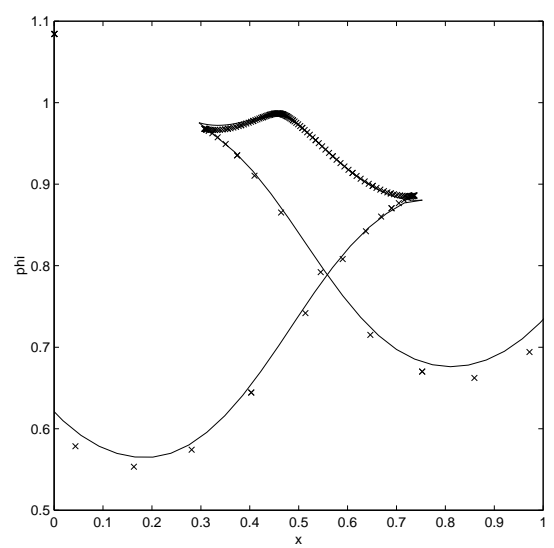

Figure 25: Phase at $s=1$.. Solid line : Eulerian. Crosses : Lagrangian. 
We believe the derivation of ODEs for the caustic curves and the closure of systems of Hamilton-Jacobi equations for the three branches to be a step forward in this field. Numerically, the delicate part of the method is the treatment of the $(s, p)$ "cusp-window". Several complex interactions of numerical parameters are at work there : the length of the time window, the $p$ support of the solution, the $X$ space discretization... Finally even though we realized a reasonable number of numerical tests, we must say that the convergence and stability of the numerical techniques presented in section 5 have not yet been investigated.

We plan next to use this Eulerian algorithm as the building block of a more general method capable of solving problems exhibiting and arbitrary number of cusps (possibly interacting). A first step in this direction may be found in [8]. This would solve the 2-D problem.

While it is still fairly easy to deal with rays in 2-D, the difficulty increases dramatically in 3-D where caustics are now surfaces. This is the real challenge for Eulerian method. The numerical techniques presented in section 5 can be extended in higher dimensions and should work for the "simple" case of folds and cusps extended in 3-D. In 3-D however, we must consider three additional generic structures for the caustics associated to a parametric representation using polynomials of degree 4 . We hope that the methodology developed in this paper will permit to analyze and solve these cases.

\section{Acknowledgments}

The method presented in this paper was partly motivated by an on-going project which involves Olivier Lafitte (CEA), Rémi Sentis (CEA) and Ian Solliec (INRIA). Let these people be thanked for their comments and suggestions on this work.

\section{References}

[1] R. Abgrall. Numerical Discretization of First Order Hamilton Jacobi Equations on Triangular Meshes. Comm. in Pure and Applied Math., 1996.

[2] R. Abgrall and J.-D. Benamou. Big ray tracing and eikonal solver on unstructured grids : Application to the computation of a multi-valued travel-time field in the marmousi model. Geophysics, 64:230239.

[3] V. I. Arnol'd. Mathematical methods of Classical Mechanics. Springer-Verlag, 1978.

[4] V. I. Arnol'd. Catastrophe theory. Springer-Verlag, 1992.

[5] V. I. Arnol'd, S.M. Gusein-Zade, and A.N. Varchenko. Singularities of Differential Maps. Birkhauser, 1986.

[6] G. Barles. Solutions de viscosité des équations de Hamilton-Jacobi. Springer-Verlag, 1994.

[7] J.-D. Benamou. Big ray tracing : Multi-valued travel time field computation using viscosity solutions of the eikonal equation. J. Comp. Physics, 128:463-474, 1996.

[8] J.-D. Benamou. Direct solution of multi-valued phase-space solutions for hamilton-jacobi equations. Comm. Pure Appl. Math., 52, 1999.

[9] J.-D. Benamou and Ph. Montarnal. Equations "géométriques" pour les calcul d'amplitudes d'ondes haute fréquence. preprint, 1999.

ESAIM: Proc., Vol. 11, 2002, 13-40 
[10] J.-D. Benamou and I. Solliec. An eulerian method for capturing caustics. J. Comp. Physics, to appear.

[11] M. Berry. Rays, wavefronts and phase: a picture book of cusps. In H.K. Kuiken H. Blok, H.A. Fewerda, editor, Huygens' Principle 1690-1990 : Theory and Applications. Elsevier, 1992.

[12] G. Beylkin. Imaging of discontinuities in the inverse scattering problem by inversion of a causal generalized radon transform. Journal of Mathematical Physics, 26:99-108, 1985.

[13] N. Bleistein and S. H. Gray. An extension of the born inversion method to a depth dependent reference profile. Geophysical Prospecting, 33:999-1022, 1985.

[14] Y. Brenier and L.Corrias. A kinetic formulation for multi-branch entropy solutions of scalar conservation laws. Ann. IHP Analyse non-linéaire, 1996.

[15] M.G. Crandall and P.L. Lions. Viscosity solutions of hamilton-jacobi equations. Trans. Amer. Math. Soc., 277:1-42, 1983.

[16] M.G. Crandall and P.L. Lions. Two approximation solutions of hamilton-jacobi equations. Math. Comp., 43:1-19, 1984.

[17] J.J. Duistermaat. Oscillatory integrals, lagrange immersions and unfolding of singularities. Comm. Pure Appl. Math., 27:207-281, 1974.

[18] B. Engquist, E. Fatemi, and S. Osher. Numerical resolution of the high frequency asymptotic expansion of the scalar wave equation. J. Comp. Physics, 120:145-155, 1995.

[19] B. Engquist and O. Runborg. Multi-phase computation in geometrical optics. Tech report, Nada KTH, 1995.

[20] Ph. Montarnal F. Golse, O. Lafitte and R. Sentis. Sur la simulation numérique de la propagation laser. Rapport CEA, 1999.

[21] M. V. Fedoryuk. Partial Differential Equations (Chap. 1). Springer-Verlag, 1988.

[22] I.M. Gelfand and S.V Fomin. Calculus of Variation. Prentice-Hall, 1963.

[23] S. Izumiya. The theory of legendrian unfoldings and first order differential equations. Proc. Royal Soc. Edinburgh, 123A:517-532, 1993.

[24] M. Fang J. Steinhoff and L. Wang. A new eulerian method for the computation of propagating short acoustic and electromagnetic pulses. J. Comp. Physics, to appear.

[25] J.B. Keller. A geometrical theory of diffraction. In Calculus of variations and its applications Vol, 8. McGraw-Hill, New-York, 1958.

[26] G. Lambare, P. Lucio, and A. Hanyga. Two dimensional multi-valued traveltime and amplitude maps by uniform sampling of a ray field. Geophys. J. Int, 125:584-598, 1996.

[27] D. Ludwig. Uniform asymptotic expansions at a caustic. Comm. Pure Appl. Math., 19:215-250, 1966.

[28] S. J. Osher and C.W. Shu. High-order essentially nonoscillatory schemes for hamilton-jacobi equations. SIAM J. Numer. Anal., 83:32-78, 1989.

[29] B. Merryman S. Ruuth and S.J. Osher. A fixed grid method for capturing the motion of selfintersecting interfaces and related pdes. preprint, 1999.

[30] P. E. Souganidis. Approximation schemes for hamilton-jacobi equations. J. Differential Equations, 59:1-43, 1985. 
[31] W. Symes. A slowness matching algorithm for multiple traveltimes. TRIP report, 1996.

[32] W. Symes and J.L. Quian. An adaptive finite difference method for traveltime and amplitudes. TRIP Report Rice U., 1999.

[33] W. Symes, R. Versteeg, A. Sei, and Q. H. Tran. Kirchhoff simulation migration and inversion using finite-difference traveltimes and amplitudes. TRIP tech. Report, Rice U., 1994.

[34] J. Van Trier and W. W. Symes. Upwind finite-difference calculation of traveltimes. Geophysics, $56: 812-821,1991$.

[35] V. Vinje, E. Iversen, and H. Gjoystdal. Traveltime and amplitude estimation using wavefront construction. Geophysics, 58:1157-1166, 1993.

[36] L.C. Young. Lecture on the Calculus of Variation and Optimal Control Theory. 1969.

Jean-David Benamou

INRIA-Rocquencourt

B.P. 105

F-78153 Le Chesnay Cedex, France

mailto:Jean-David.Benamou@inria.fr

http://www-rocq.inria.fr/ benamou 